\section{$C_{\text {chiture }}$}

- Additional material is

published online only. To view please visit the journal online (http://dx.doi.org/10.1136/ thoraxjnl-2016-208768).

${ }^{1}$ Department of Anesthesia and Intensive Care and Emergency Department, Fondazione IRCC Policlinico San Matteo, University of Pavia, Pavia, Italy ${ }^{2}$ Adult Intensive Care Unit, Royal Brompton \& Harefield NHS Foundation Trust, London, UK

${ }^{3}$ National Heart and Lung institute, Imperial College, London, UK

Correspondence to Dr Guido Tavazzi, Fondazione IRCC Policlinico San Matteo, Piazzale Golgi 19, Pavia 27100 Italy; gtavazzi@yahoo.it

Received 13 April 2016 Revised 1 November 2016 Accepted 17 November 2016 Published Online First 7 December 2016

\title{
Visual identification of pulmonary ventilation and perfusion: a new application of lung ultrasound
}

\author{
Guido Tavazzi, ${ }^{1,2}$ Francisca Ana Caetano, ${ }^{2}$ Sachin Shah, ${ }^{2}$ Joana Alcada, ${ }^{2,3}$ \\ Susanna Price ${ }^{2}$
}

A 32-year-old lady with a history of haemochromatosis with iron infiltration of the myocardium and aplastic anaemia was admitted with H1N1-related severe adult respiratory distress syndrome with dense bilateral consolidations in all lobes at chest $\mathrm{X}$-ray and CT scan (figure 1A, B). The severe hypoxaemia requiring mechanical ventilation and refractory to inhaled nitric oxide and pronation led ultimately to venovenous extracorporeal membrane oxygenation placement. Transthoracic echocardiography (TTE) demonstrated a mildly biventricular systolic impairment alike the last TTE done during the follow-up for the haemochromatosis, without any intracardiac shunt as demonstrated by agitatedsaline bubble study. Lung ultrasound (LUS) showed bilateral complete consolidation of the lung (tissuelike pattern) in the mid-lower lobes, which was present from admission. On the day 3, the decline in minute volume, worsening gas exchange and decrease of $\mathrm{V}_{\mathrm{CO} 2}$ from the native lung corresponded to the presence of arterial systolic and venous diastolic flow on colour Doppler within the consolidation as sign of worsening V/Q mismatch (figure 1C, D; see online supplementary Videos 1 and 2).
The detection of intrapulmonary shunting has been always derived from static imaging techniques, clinical assessment and interpretation of arterial blood gases. LUS is widely used as an integrative tool in the diagnosis of the underlying cause of acute respiratory failure and extent of the pathology. Protocols and guidelines have standardised and validated the nomenclature of the various patterns of alveolar-interstitial syndrome and loss of aeration. ${ }^{12}$ The 'tissue-like pattern' corresponds to consolidation with complete loss of aeration, which may be associated with a degree of V/Q mismatch, therefore intrapulmonary shunt, and corresponding hypoxaemia.

Although the relation between V/Q mismatch and intrapulmonary shunt with colour Doppler (using standard LUS protocols ${ }^{1}$ ) has not been appropriately demonstrated yet, the demonstration of pulsatile flow in non-areated lung is associated with severe consolidation and likely to be an indirect demonstration of extreme V/Q mismatch/intrapulmonary shunt, in a manner that can be monitored real-time in response to therapeutic manoeuvres including bronchoscopy, positioning and recruitment manoeuvres. ${ }^{3}$

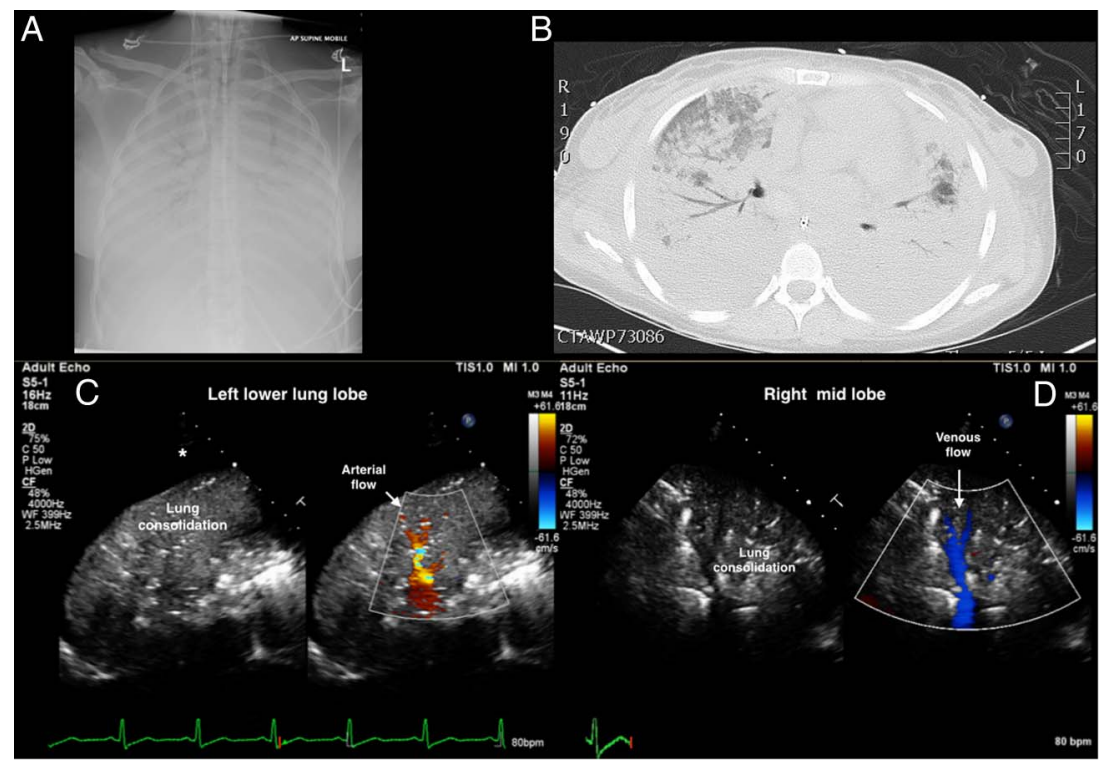

Figure 1 (A) Chest X-ray showing bilateral consolidation and air bronchogram, confirmed at CT scan (B). (C) Lung ultrasound transversal scan of the left lower lobe using the cardiac probe. Left side of the panel complete consolidation of inferior lobe (tissue-like pattern) surrounded by a small amount of pleural effusion $\left(^{*}\right)$. On the right side, colour flow Doppler technique is applied showing the arterial flow into the lung parenchyma (arrow-red flow). (D) The same technique applied to the right middle lobe and the venous flow is shown (arrow—blue flow). 
Contributors GT, FAC, SS and JA: data acquisition. GT, FAC, SS, JA, SP: image interpretation, intellectual contribution and patient treatment.

Competing interests None declared.

Patient consent Obtained.

Provenance and peer review Not commissioned; externally peer reviewed.

\section{REFERENCES}

1 Lichtenstein DA, Mezière GA. Relevance of lung ultrasound in the diagnosis of acute respiratory failure: the BLUE protocol. Chest 2008;134;117-25.

2 Volpicelli G, Elbarbary M, Blaivas M, et al. International evidence-based recommendations for point-of-care lung ultrasound. Intensive Care Med 2012;38:577-91.

3 Xirouchaki N, Kondili E, Prinianakis $\mathrm{G}$, et al. Impact of lung ultrasound on clinical decision making in critically ill patients. Intensive Care Med 2014;40:57-65. 